\title{
In vitro spectroscopic investigation of human cataract lens capsule hydropermeability
}

\author{
Andrey V. Belikov ${ }^{1}$, Alexey M. Zagorulko ${ }^{2}$, Vyacheslav I. Kochubey ${ }^{3,4}$, and Sergey N. Smirnov ${ }^{1^{*}}$ \\ ${ }^{1}$ ITMO University, 49 Kronverksky Pr., Saint Petersburg 197101, Russia \\ 2 St. Petersburg Branch of the S. Fyodorov Eye Microsurgery Federal State Institution, 21 Yaroslav Gashek St., \\ Saint Petersburg 192283, Russia \\ ${ }^{3}$ N.G. Chernyshevsky Saratov State University, 83 Astrakhanskaya St., Saratov 410012, Russia \\ ${ }^{4}$ Tomsk State University, 36 Prosp. Lenina, Tomsk 634050, Russia \\ *e-mail: s.n.smirnov@niuitmo.ru
}

\begin{abstract}
The dynamics of collimated transmission of optical radiation in the wavelength range 1100-2000 $\mathrm{nm}$ during the in vitro dehydration of human cataract lens capsules in air was studied for the first time. For capsules separated from human cataract lenses the average value of the hydropermeability coefficient at dehydration was $0.19 \pm 0.10 \mu \mathrm{m} / \mathrm{s}$. The average characteristic time of cataract lens capsules dehydration was $77 \pm 10 \mathrm{~s}$ for the samples studied. (C) 2017 Journal of Biomedical Photonics \& Engineering.
\end{abstract}

Keywords: cataract; eye lens; capsule; capsulorhexis; transmission; laser; water; hydropermeability.

Paper \#3214 received 5 Jun 2017; revised manuscript received 14 Sep 2017; accepted for publication 15 Sep 2017; published online 30 Sep 2017. doi: 10.18287/JBPE17.03.030303.

\section{References}

1. B. P. Danysh, and M. K. Duncan, "The lens capsule," Experimental Eye Research 88(2), 151-164 (2009).

2. K. Jeon, International review of cell and molecular biology, Volume 296, Academic Press (2012). ISBN: 9780123943071.

3. J. S. Friedenwald, "Permeability of the lens capsule: with special reference to the etiology of senile cataract," Archives of Ophthalmology 3(2), 182-193 (1930).

4. J. S. Friedenwald, "The permeability of the lens capsule to water, dextrose and other sugars," Transactions of the American Ophthalmological Society 28, 195-211 (1930).

5. I. G. Fels, "Permeability of the anterior bovine lens capsule," Experimental Eye Research 10(1), 8-14 (1970).

6. L. Ozaki, "The barrier function of the posterior capsule," American Intra-Ocular Implant Society Journal 10(2), 182-184 (1984)

7. R. F. Fisher, "The water permeability of basement membrane under increasing pressure: evidence for a new theory of permeability," Proceedings of the Royal Society B: Biological Sciences 216(1205), 475-496 (1982).

8. K. Varadaraj, C. Kushmerick, G. J. Baldo, S. Bassnett, A. Shiels, and R. T. Mathias, "The Role of MIP in Lens Fiber Cell Membrane Transport,” Journal of Membrane Biology 170(3), 191-203 (1999).

9. K. Varadaraj, S. Kumari, A. Shiels, and R. T. Mathias, "Regulation of Aquaporin Water Permeability in the Lens," Investigative Ophthalmology \& Visual Science 46(4), 1393-1402 (2005).

10. K. Varadaraj, S. S. Kumari, and R. T. Mathias, "Functional Expression of Aquaporins in Embryonic, Postnatal, and Adult Mouse Lenses," Developmental Dynamics 236(5), 1319-1328 (2007).

11. C. J. Lee, J. A. Vroom, H. A. Fishman, S. F. Bent, "Determination of human lens capsule permeability and its feasibility as a replacement for Bruch's membrane," Biomaterials 27(8), 1670-1678 (2006).

12. B. P. Danysh, T. P. Patel, K. J. Czymmek, D. A. Edwards, L. Wang, J. Pande, and M. K. Duncan, "Characterizing molecular diffusion in the lens capsule," Matrix Biology 29(3), 228-236 (2010).

13. C. Kastner, M. Lubler, T. Reske, K. Sternberg, R. Guthoff, and K.-P. Schmitz, "Determination of human anterior lens capsule permeability for fluorescent model substances and after-cataract preventive drugs," Biomedical Engineering / Biomedizinische Technik, 57(SI-1 Track-D), 561-563 (2012). 
14. K. E. Donaldson, R. Braga-Mele, F. Cabot, R. Davidson, D. K. Dhaliwal, R. Hamilton, M. Jackson, L. Patterson, K. Stonecipher, and S. H. Yoo, "Femtosecond laser-assisted cataract surgery," Journal of Cataract \& Refractive Surgery 39(11), 1753-1763 (2013).

15. Z. Z. Nagy, "The role of femtolaser in cataract surgery," European Ophthalmic Review 6(5), 286-289 (2012).

16. V. G. Kopayeva, S. U. Kopayev, A. A. Ginoyan, and V. U. Alborova, "Clinical experience in laser cataract extraction," Bulletin of the Russian Academy of Natural Sciences 1, 77-80 (2012) [In Russian].

17. S. N. Fyodorov, V. G. Kopaeva, Yu. V. Andreev, A. V. Erofeev, A. V. Belikov, E G. Bogdalova, A. V. Skripnik, and O. A. Frolova, "Laser Extraction of Cataract (Experimental studies)," Ophtalmosurgery 3, 3-10 (1998) [in Russian].

18. N. J. Friedman, D. V. Palanker, G. Schuele, D. Andersen, G. Marcellino, B. S. Seibel, J. Batlle, R. Feliz, J. H. Talamo, M. S. Blumenkranz, and W. W. Culbertson, "Femtosecond laser capsulotomy," Journal of Cataract \& Refractive Surgery 37(7), 1189-1198 (2011).

19. A. V. Belikov, S. V. Gagarsky, A. B. Gubin, S. Ya. Weiner, A. N. Sergeev, and S. N. Smirnov, "Subjoule diode-pumped ytterbium-erbium glass laser with cavity dumping for cataract extraction," Scientific and Technical Journal of Information Technologies, Mechanics and Optics 15(6), 1021-1029 (2015).

20. A. V. Belikov, S. V. Gagarsky, A. N. Sergeev, and S. N. Smirnov, "In vitro destruction of anterior human lens capsule by submicrosecond pulses of Yb,Er:Glass laser," Proc. SPIE 10336, 103360B (2017).

21. B. S. Ross, and C. A. Puliafito, "Erbium-YAG and Holmium-YAG Laser Ablation of the Lens," Lasers in Surgery and Medicine 15(1), 74-82 (1994).

22. H. Ho, and E. Fischer, "Pilot Study on Erbium Laser Phacoemulsification," Ophthalmology 107(6), 10531061 (2000).

23. F. Hausladen, H. Wurm, and K. Stock, "Basic studies on laser-assisted phacoemulsification using diodepumped Er:YAG laser," Proc. SPIE 9693, 96931Y (2016).

24. K. F. Palmer, and D. Williams, "Optical properties of water in the near infrared," Journal of the Optical Society of America 64(8), 1107-1110 (1974).

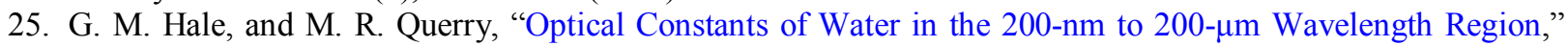
Applied Optics 12(3), 555-563 (1973).

26. A. N. Bashkatov, E. A. Genina, Yu. P. Sinichkin, V. I. Kochubey, N. A. Lakodina, and V. V. Tuchin, "Glucose and Mannitol Diffusion in Human Dura Mater," Biophysical Journal 85(5), 3310-3318 (2003).

27. D. K. Tuchina, R. Shi, A. N. Bashkatov, E. A. Genina, D. Zhu, Q. Luo, and V. V. Tuchin "Ex vivo optical measurements of glucose diffusion kinetics in native and diabetic mouse skin," Journal of Biophotonics 8(4), 332-346 (2015).

28. D. K. Tuchina, A. N. Bashkatov, E. A. Genina, and V. V. Tuchin, "Quantification of glucose and glycerol diffusion in myocardium," Journal Innovative Optical Health Science 8(3), 1541006 (2015).

29. E. A. Genina, A. N. Bashkatov, Yu. P. Sinichkin, I. Yu. Yanina, and V. V. Tuchin, "Optical clearing of biological tissues: Prospects of application in medical diagnostics and phototherapy [Review], Journal of Biomedical Science \& Engineering 1(1), 22-58 (2015).

30. D. K. Tuchina, V. D. Genin, A. N. Bashkatov, E. A. Genina, and V. V. Tuchin, "Optical Clearing of Skin Tissue ex vivo with Polyethylene Glycol," Optics and spectroscopy 120(1), 28-37 (2016).

31. A. N. Bashkatov, "Control of tissue optical properties by means of osmotically active immersion liquids," PhD thesis, Saratov State University, Saratov, Russia (2002) [In Russian].

32. E. A. Genina, A. N. Bashkatov, A. A. Korobko, E. A. Zubkova, V. V. Tuchin, I. Yaroslavsky, and G. B. Altshuler, "Optical clearing of human skin: comparative study of permeability and dehydration of intact and photothermally perforated skin,” Journal of Biomedical Optics 13(2), 021102 (2008).

33. A. Kotyk and K. Janáček, Membrane Transport: An Interdisciplinary Approach, Springer US, Plenum Press, New York (1977), ISBN: 978-1-4684-3335-7.

\section{Introduction}

The lens of the eye does not contain blood vessels; the only filter for the transit of water, nutrients, and products of vital activity is the membrane in which it is housed - the lens capsule. The lens capsule is a thin structureless transparent membrane which completely covers the lens. A part of the capsule that lines the anterior surface of the eye lens is called the anterior lens capsule. From the inside, the anterior capsule is covered with a single-layered epithelium. The devoid of epithelium posterior capsule is almost twice thinner than the anterior. The lens capsule is a matrix of molecules consisting mainly of type IV collagen and laminin networks, which are bound together by nidogen and perlecan [1]. It represents one of the varieties of basement membranes, and is the thickest human basement membrane [2].

Since the viability of the lens is provided by the capsule, interest in the problem of determining the permeability of a given membrane to various substances appeared very long ago. One of the first works on determination of the relative permeability of the lens 
capsule to water and various sugars date back to 1930 $[3,4]$.

In [5] the hydrodynamic permeability of the bovine lens capsule to water and the osmotic permeability to glucose were studied. For the investigation of permeability the authors used a device, which represented a U-shaped tube formed by a reservoir $\left(V=700 \mathrm{~cm}^{3}\right)$ and a thin capillary $(d=0.1 \mathrm{~mm})$ between which a sample (capsule) was fixed. The volumetric flow was determined from the magnitude of the height of liquid in the capillary. The need for the fixation of the capsule between two reservoirs (similar constructions were also used in [6-7]), increases the sensitivity of such method to integrity and requires a sufficiently large size of the lens capsule samples. It should be also noted that permeability measurements were carried out under hydrostatic pressure.

In [8-10] the fundamental properties of integral membrane proteins forming pores in membranes of cells-aquaporin's, were studied, their role in the transport of substances through the capsule was investigated. The hydropermeability coefficients of anterior epithelium cells and vesicles of cell membranes of the animal lens fibers were measured.

In [11] the anterior lens capsule of the human eye was examined as a potential replacement for the Bruch membrane for the treatment of age-related macular degeneration. One of the most important properties of the Bruch membrane is the ensuring of a flow of nutrients and products of vital activity between the retinal pigment epithelium and the choriocapillaries. In this connection, the authors measured the lens capsule permeability by studying the diffusion of FITC-dextran molecules (fluorescein isothiocyanate-dextran) with different molecular masses. Diffusion of dextran molecules was performed in a blind-well chamber and the concentration of diffused molecules was measured by recording the fluorescence intensity of the solution.

The studies of the lens capsule permeability, as well as the development of new methods for its measurement, are still relevant. In [12] a method for studying the lens capsule permeability based on the restoration of fluorescence after photobleaching was demonstrated. In [13] the possibility of preventing the occurrence of secondary cataracts by introducing drugs into an artificial intraocular lens was investigated. In this connection, the permeability coefficients of the lens capsule to antiproliferative drugs were measured. The measurements were carried out by the fluorescence detection method, molecular weight cutoff was also measured.

The data on the lens capsule permeability are also of interest for modern ophthalmic surgery, also due to the fact that minimally invasive laser technologies for cataract surgery gain ground. The most commonly used minimally invasive method of cataract surgery is ultrasonic phacoemulsification. Recently, the technology of femtosecond laser-assisted ultrasound phacoemulsification has been actively introduced into surgical practice. In this case femtosecond laser devices are used for opening the anterior lens capsule (capsulorhexis) and pre-softening dense cataract lens [14], but for complete lens fragmentation the additional ultrasonic machine is required. Now, white, brown and black cataracts [15], as well as complications with weak ciliary zonulas [16], are limitations for the application of femtosecond laser-assisted ultrasound phacoemulsification. In ophthalmological clinics in Russia and abroad, along with ultrasonic and femtosecond phacoemulsification, the method of laser cataract extraction (LCE) [17] based on the use of $\mathrm{Nd}$ :YAG laser radiation with the wavelength of $1444 \mathrm{~nm}$ is widely used. The LCE method is based on the destruction of cataract lenses due to the complex effect of laser radiation and laser-induced acoustic waves. The main advantage of this method is the ability to destruct cataract lenses of any density degree without negative influence on surrounding issues. The lens destruction process is characterized by high productivity - time of complete destruction is of 2-3 minutes [16].

An important stage of minimally invasive cataract surgery, including LEC, is anterior capsulorhexis opening of the anterior cataract lens capsule. The more precisely capsulorhexis is performed, the more reliable is the fixation of the artificial intraocular lens (IOL), which is installed in the capsular bag at the final stage of the operation as a replacement for the removed cataract lens. Currently, capsulorhexis during LEC is performed either manually, or with the help of complex and expensive femtosecond laser systems. Such laser systems provide a cut of the capsule with the shape of an ideal circle with a given diameter, the edge of the capsule is characterized by greater strength than in the case of manual capsulorhexis [18]. In recent times, the use of a microsecond $\mathrm{Yb}$,Er:Glass laser radiation with a wavelength of $1.54 \mu \mathrm{m}$ has been discussed [19] for efficient fragmentation of the cataract lens and for performing anterior capsulorhexis [20]. On the other hand, for performing a capsulorhexis it is also possible to use the radiation of lasers on crystalline media activated by erbium ions; the use of Er:YAG laser radiation for lens fragmentation was investigated in [21-23].The radiation of Er:YAG laser with a wavelength of $2.94 \mu \mathrm{m}$ is very effectively absorbed by water [24,25]; as a result, the penetration depth of this radiation into water-saturated tissues, to which the lens capsule can be attributed, is close to $1 \mu \mathrm{m}$. It is obvious that in the process of laser capsulorhexis, during the action of the radiation pulse, the capsule is dehydrated, and in a pause between laser pulses it is saturated with water again. In this regard, in order to select the energy and time parameters of laser radiation for efficient and safe impact on the cataract lens capsule it is important to know how quickly the part of the capsule dehydrated under the action of laser radiation can be saturated with water. The hydropermeability of cataract lens capsules is poorly understood.

Thus, the study of hydropermeability of human cataract lens capsules with the use of effective, non- 
contact techniques, including optical techniques, is important and relevant for the development of modern methods of minimally invasive laser cataract surgery. From this point of view, a method for measuring the diffusion properties of biological tissues described in [26-31] is interesting, which is effectively used to study the optical clearing of biological tissues under the action of immersion agents. The method is based on obtaining data on the characteristic times and coefficients of diffusion as well as on the permeability of membranes for osmotically active liquids from data on the dynamics of collimated transmission of optical radiation by samples of biological tissues under the influence of these liquids.

In the present study, the dynamics of collimated transmission of optical radiation in the wavelength range 1100-2000 $\mathrm{nm}$ for cataract lens capsules in vitro during their dehydration in air was studied for the first time. The analysis of the optical density dynamics made it possible to determine the coefficient of hydropermeability and the characteristic time of dehydration of the human cataract lens capsule.

\section{Materials and methods}

The samples of human lens capsules were obtained by performing manual capsulorhexis during LEC, after extraction they were stored in saline $(0.9 \%$ aqueous sodium chloride solution) at a temperature of $4-6{ }^{\circ} \mathrm{C}$. In total, five capsules of medium-density human cataract lenses were examined. To measure the thickness of lens capsules the samples were placed between the coverslips; the measurements were carried out with a micrometer "S_Mike PRO" ("Sylvac", Switzerland).

The determination of the hydropermeability coefficient (dehydration rate) and the characteristic time of dehydration of the cataract lens capsule was carried out on the basis of studying the dynamics of optical signal attenuation associated with the absorption of radiation by the liquid during the dehydration of the capsule in air. This study was carried out in the same way as described in [26-31], where the diffusion of various osmotically active liquids in biological tissues was investigated.

The lens capsule sample was placed onto a glass slide. After that the liquid (saline solution) remaining on the capsule surface was removed mechanically by the lateral surface of injection needle which was moved along the capsule surface. This action was implemented with the minimum possible pressure from the needle side to the capsule sample.

Then a sample of the capsule on the glass slide was placed in the measuring channel of the experimental setup (Fig. 1). The experimental setup included a radiation source (1) - a halogen lamp "HL 2000" ("Ocean Optics", USA), light guides "QP600-2-VISNIR" ("Ocean Optics", USA) $(2,5)$, one of which delivered radiation to the sample (4), the other collected the radiation transmitted through the sample, a diaphragm (3) with a diameter of $1 \mathrm{~mm}$, a spectrometer (6) "NIR Quest" ("Ocean Optics", USA) and a computer (7).

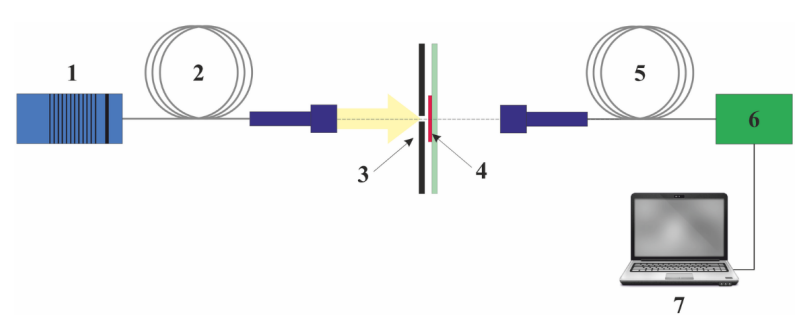

Fig. 1 Scheme of experimental setup for collimated transmission measurement: 1 - halogen lamp "HL 2000" ("Ocean Optics", USA); 2,5 - light guides "QP600-2-VIS-NIR" ("Ocean Optics", USA) with collimators "74-ACR" ("Ocean Optics", USA) at the ends; 3 - diaphragm; 4 - sample on the glass slide; 6 - spectrometer "NIR Quest" ("Ocean Optics", USA), 7 - computer.

In experiment collimated transmission spectra of each sample of lens capsule on a glass slide $T_{\text {glass }+ \text { sample }}(\lambda, t)$ and also spectrum of glass slide $T_{\text {glass }}(\lambda)$ were measured in spectral range $1100-2000 \mathrm{~nm}$. The measurements of $T_{\text {glass }+ \text { sample }}(\lambda, t)$ were carried out for the first 3 minutes in 0.5 minute increments, then on $4^{\text {th }}, 6^{\text {th }}$ and $10^{\text {th }}$ minute, at room temperature and $20 \%$ relative humidity.

The transmission spectra of lens capsule samples $T_{\text {sample }}(\lambda, t)$ were obtained by correcting the spectra $T_{\text {glass }+ \text { sample }}(\lambda, t)$ taking into account the absorption of the glass slide and normalization to a transmission value at $1100 \mathrm{~nm}\left(T_{\text {sample }}(\lambda=1100 \mathrm{~nm}, t)\right)$ :

$$
T_{\text {sample }}(\lambda, t)=\frac{T_{\text {glass }+ \text { sample }}(\lambda, t)}{T_{\text {glass }}(\lambda) T_{\text {glass }+ \text { sample }}(\lambda=1100 \mathrm{~nm}, t)} .
$$

The wavelength of $1100 \mathrm{~nm}$ was chosen as the reference one under the assumption that the scattering coefficients for a transparent capsule are small and close to each other for the investigated spectral range, and the absorption of water at a given wavelength is negligible (Fig. 2).

A baseline drift of the measuring device causes the need for such a normalization during the experiment. The baseline drift may be connected with the time variation of the refractive index of the sample (due to dehydration) when the glass slide with the sample is not perpendicular to the light beam. The baseline drift may be also associated with incompletely aligned sphericity of the sample when placed onto the glass slide, which leads to shift of the beam relative to the optical axis. The normalized collimated transmission spectra were averaged over five samples, after that the spectral dependences of the average optical density were obtained from the average transmission spectra: 


$$
D(\lambda, t)=\lg \left(\frac{1}{T_{\text {sample }}(\lambda, t)}\right)
$$

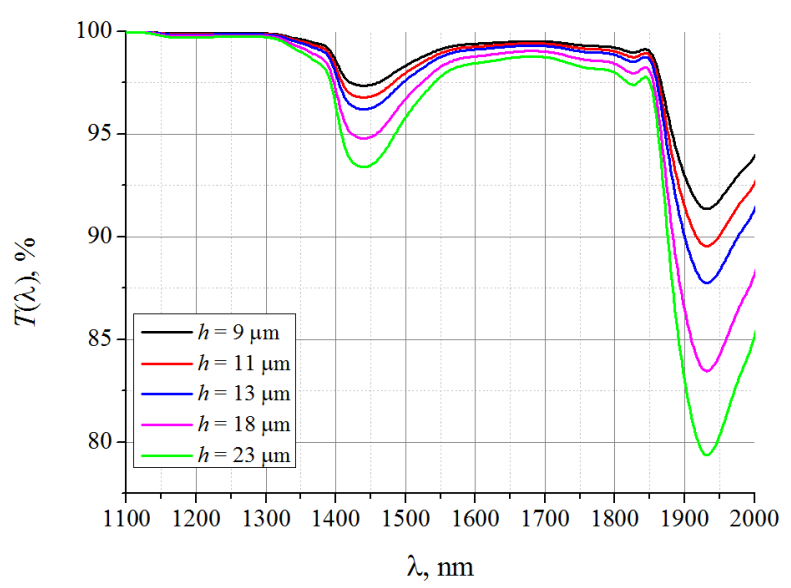

Fig. 2 Transmission spectra $T(\lambda)$ of water layers of thickness $h$ equal to thickness of investigated lens capsule samples.

The time variation in the optical density of lens capsules at the wavelength of $1950 \mathrm{~nm}$, which align with the local absorption peak of water contained in the lens capsule, is associated with the dehydration of the capsule. Dehydration, in turn, is associated with the diffusion of water from the sample into the external volume. In this connection, the dynamics of the dehydration degree change can be described using the equation for diffusion through the permeable membrane. For estimation of dehydration degree under the action of air, the following equation can be used [30]:

$$
\frac{C(t)}{C_{0}}=\frac{M(t)-M_{r e s}}{M(t=0)-M_{r e s}}=\exp \left(-\frac{t}{\tau_{D}}\right),
$$

where $M$ is the mass of the biotissue sample, $M_{\text {res }}$ is the residual mass of the sample (i.e., completely dehydrated), $C$ is the concentration of the diffusing substance, $C_{0}$ is the concentration of the substance in the closed volume at the initial time, $\tau_{D}$ is the time constant characterizing the dehydration rate and $t$ is the dehydration time.

The parameter $M_{\text {res }}$ includes the mass of collagen, elastin and other components of the tissue after complete dehydration, bound water and possible residual free water. The difference between the initial and residual mass of the sample $\left(M(t=0)-M_{\text {res }}\right)$ indicates the amount of water which left the tissue due to dehydration.

Eq. (3) is similar in shape to the diffusion equation, which describes the flow of substance from a small volume with a nonzero concentration to a tank of zero concentration [31]:

$$
C(t)=C_{0} \exp \left(-\frac{P S}{V} t\right)
$$

where $C_{0}$ is the concentration of substance in a closed volume at the initial time, $P$ is the permeability coefficient, $S$ is the area of the membrane and $V$ is the closed volume.

The permeability coefficient $P$ of membrane in the case of one-sided diffusion is related to the characteristic diffusion time $\tau_{D}$ of water in the capsule by the expression:

$$
P=\frac{h}{\tau_{D}}
$$

where $h$ is the membrane thickness.

Since the optical density of the lens capsule at the wavelength of $1950 \mathrm{~nm}$ is directly proportional to the concentration of water contained in it, taking the sample thickness unchanged during the measurements, the dynamics of the sample optical density change $D(t)$ during its dehydration by analogy with equation (3) can be described by the following expression

$$
D(t)=\left(D(t=0)-D_{\text {res }}\right) \exp \left(-\frac{t}{\tau_{D}}\right)+D_{\text {res }},
$$

where $t$ is the dehydration time, $\tau_{D}$ is the time constant characterizing the dehydration rate, $D_{\text {res }}$ is the residual optical density associated with the absorption of radiation by a dry matter and possible residual water.

Thus, the average for the samples studied characteristic dehydration time of human cataract lens capsules (the characteristic water diffusion time $\tau_{D}$ in the capsule) can be obtained by approximating the experimentally obtained points of the time dependence of the optical density of the samples at the wavelength of $1950 \mathrm{~nm}$.

In our study, the approximation of the experimental data was carried out by using non-linear fitting with final value fixation in the package "OriginPro 8.5.1" (OriginLab Corp., USA). The following standard exponential function was used

$$
y(x)=A \exp (B x)+C
$$

The program generated the values of approximation coefficients and corresponding values of standard deviation.

\section{Results and discussion}

Averaged over all samples spectra of optical density at different times are shown in Fig. 3a. Figure $3 b$ shows the experimentally obtained values of optical density of the lens capsules at the wavelength of $1950 \mathrm{~nm}$ at different times and the results of the approximation of 
these values by the function (7). It is seen that in the first 3 minutes the optical density at the wavelength of $1950 \mathrm{~nm}$ drops fast, then a segment of slower bleaching is observed.
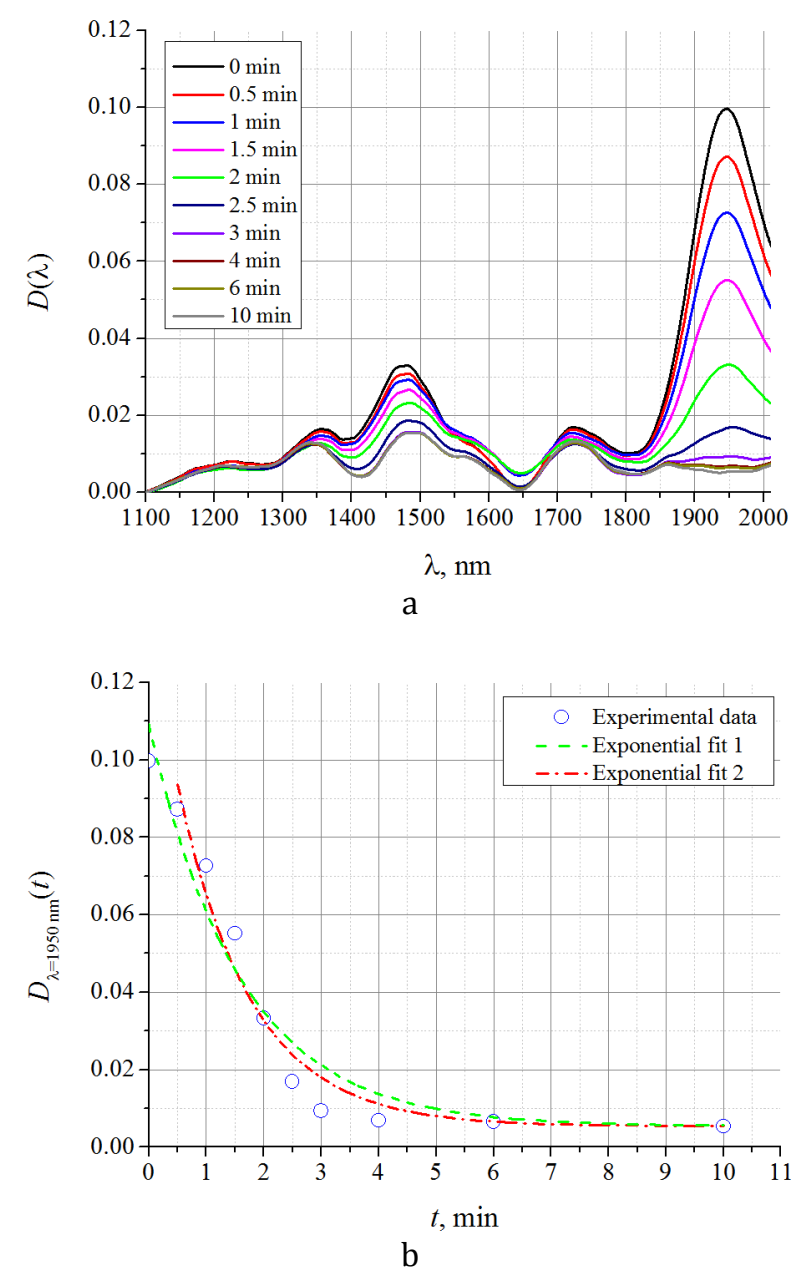

Fig. 3 Lens capsule optical density spectra $D(\lambda)$ obtained according Eq. (2) in different time points (a); dependence of lens capsule optical density $D_{\lambda=1950 \mathrm{~nm}}(t)$ at the wavelength of $1950 \mathrm{~nm}$ on dehydration time (b).

The accuracy of the result can be affected by the fact that the thickness of the sample as decreases during the dehydration (drying). The point $D(t=0)$ can introduce an error in the result of approximation due to a inconstancy of the time interval between the moment of setting the glass slide with the sample into the measuring channel and the moment of the beginning of transmission spectrum measurement. In this connection, in Fig. $3 b$ two versions of the experimental points approximation are presented, taking into account the point $D(t=0)$ ("Exponential fit 1") and with its exception ("Exponential fit 2").

As a result of the "Exponential fit 2" approximation the mean over samples studied value of characteristic time of dehydration (diffusion of water from the sample) of the anterior cataract lens capsule was obtained: $\tau_{D}=77 \pm 10 \mathrm{~s}$. By using "Exponential fit 1", this value was $95 \pm 12 \mathrm{~s}$. In our opinion, the result obtained with "Exponential fit 2" with the exception of the point $D(t=0)$ is more adequate.

The indicated error in the result is an error of approximation. At an average of five capsule thicknesses of $h=14.8 \pm 5.7 \mu \mathrm{m}$ and a dehydration time of $\tau_{D}=77 \pm 10 \mathrm{~s}$, the average value of the permeability coefficient $P$ calculated according to Eq. (5) is $P=0.19 \pm 0.10 \mu \mathrm{m} / \mathrm{s}$.

In our experiment, the capsules were not cleared from the anterior lens epithelial cells, so the latter had an effect on the permeability. It should be noted that the obtained value of the permeability coefficient of cataract lens capsule is two orders of magnitude less than the values of permeability coefficients of anterior lens epithelial cells, presented in [8, 10]. In [3] a significant decrease in the permeability of the lens capsule in the case of cataract was noted, so the observed difference in results can be explained by the fact that in our case capsules separated from the human cataract lenses were examined. It is also worth noting the fact that in our case the dehydration of the capsule took place in the air, while in $[8,10]$ the shrinking of cells caused by the change of saline osmolality from $240 \mathrm{mM}$ to $480 \mathrm{mM}$ was analyzed.

A comparison of the obtained results with the data on the lens capsule permeability coefficients obtained in [3-7] presents difficulties, because in these studies the measurements were carried out in the presence of hydrostatic pressure.

If we assume that the width of the dehydrated tissue zone near the boundary of laser-produced microperforation in capsule is comparable to the penetration depth of laser radiation, then for lasers on crystalline media activated by erbium ions (for example, Er:YAG laser), it amounts to about $1 \mu \mathrm{m}$. Taking into account the hydropermeability coefficient measured in this paper, this zone will restore the initial water content, and hence the initial absorption coefficient, only on average $5 \mathrm{~s}$ after laser exposure. Thus, for effective capsulorhexis by means of erbium laser radiation this feature should be taken into account when choosing a technology for scanning a laser beam over the surface of the capsule.

\section{Conclusion}

The paper describes a method for measuring the hydropermeability of the lens capsule and the characteristic time of its dehydration in air using optical spectroscopy. For capsules separated from the cataract lenses of the human eye the average over studied samples value of hydropermeability coefficient was $0.19 \pm 0.10 \mu \mathrm{m} / \mathrm{s}$. The average characteristic time of dehydration was $77 \pm 10 \mathrm{~s}$. The method can be used for express studies of lens capsule samples in order to obtain information about their physical condition. The results obtained with the help of this method are useful when tuning laser sources for capsulorhexis in laboratory for choosing optimal radiation parameters. The data on lens capsule hydropermeability indicate a possibility of cataract development; therefore, the 
method can also be used for studies of the influence of various drugs on eye lens. The determination of the possibility of using such method in clinical practice requires additional research and the development of special equipment.

\section{Disclosures}

The authors declare that there are no conflicts of interest related to this article.

\section{Acknowledgments}

The work was carried out under the partial financial support from the Russian Foundation for Basic Research, grant No. 16-32-50164. 